\title{
Clinical utility of the cogstate brief battery in identifying cognitive impairment in mild cognitive impairment and Alzheimer's disease
}

\author{
Paul Maruff ${ }^{1,2^{*}}$, Yen Ying Lim ${ }^{1}$ David Darby ${ }^{1}$, Kathryn A Ellis ${ }^{1,3,4}$, Robert H Pietrzak ${ }^{5}$, Peter J Snyder ${ }^{6}$, Ashley I Bush ${ }^{1}$, \\ Cassandra Szoeke ${ }^{1,4,7}$, Adrian Schembri ${ }^{2}$, David Ames ${ }^{3,4}$, Colin L Masters ${ }^{1}$ and for the AIBL Research Group
}

\begin{abstract}
Background: Previous studies have demonstrated the utility and sensitivity of the CogState Brief Battery (CBB) in detecting cognitive impairment in Alzheimer's disease (AD) and mild cognitive impairment (MCI) and in assessing cognitive changes in the preclinical stages of AD. Thus, the CBB may be a useful screening tool to assist in the management of cognitive function in clinical settings. In this study, we aimed to determine the utility of the CBB in identifying the nature and magnitude of cognitive impairments in $\mathrm{MCl}$ and $\mathrm{AD}$.

Methods: Healthy adults $(n=653)$ adults with amnestic $M C I(n=107)$, and adults with $A D(n=44)$ who completed the CBB participated in this study. Composite Psychomotor/Attention and Learning/Working Memory scores were computed from the individual CBB tests. Differences in composite scores were then examined between the three groups; and sensitivity and specificity analyses were conducted to determine cut scores for the composite scores that were optimal in identifying $\mathrm{MCl}$ - and AD-related cognitive impairment.

Results: Large magnitude impairments in $\mathrm{MCl}(\mathrm{g}=2.2)$ and $\mathrm{AD}(\mathrm{g}=3.3)$ were identified for the learning/working memory composite, and smaller impairments were observed for the attention/psychomotor composite $(\mathrm{g}$ 's $=0.5$ and 1, respectively). The cut-score associated with optimal sensitivity and specificity in identifying MCl-related cognitive impairment on the learning/working memory composite was -1SD, and in the AD group, this optimal value was $-1.7 \mathrm{SD}$. Both composite scores showed high test-retest reliability $(r=0.95)$ over four months. Poorer performance on the memory composite was also associated with worse performance on the Mini Mental State Exam and increasing severity on the Clinical Dementia Rating Scale sum of boxes score.
\end{abstract}

Conclusions: Results of this study suggest that the CogState learning/working memory composite score is reduced significantly in $\mathrm{Cl}$ and $\mathrm{AD}$, correlate well with measures of disease classification and are useful in identifying memory impairment related to $\mathrm{MCl}$ - and $\mathrm{AD}$.

\section{Background}

The importance of screening for dementia in individuals at risk of neurodegenerative diseases is now widely accepted (Snyder 2013). While advances in neuroimaging and fluid biomarkers show much promise for identifying early Alzheimer's disease (AD), neuropsychological testing remains the cornerstone of early disease recognition (Albert et al. 2011; McKhann et al. 2011). Unfortunately,

\footnotetext{
* Correspondence: pmaruff@unimelb.edu.au

'The Florey Institute of Neuroscience and Mental Health, University of Melbourne, Parkville, Victoria, Australia

${ }^{2}$ CogState Ltd., Melbourne, Victoria, Australia

Full list of author information is available at the end of the article
}

most neuropsychological test batteries shown to be sensitive to early $\mathrm{AD}$ require substantial time and expertise for both administration and scoring and this can limit their potential for use in wide-scale screening (Fredrickson et al. 2010). While some brief bedside cognitive screening instruments (i.e. measures that require less than 30 minutes for administration) such as the Mini Mental State Examination (MMSE) (Folstein et al. 1975) and Montreal Cognitive Assessment (MoCA) (Nasreddine et al. 2005) have been shown to be useful in case finding studies of $\mathrm{AD}$ and $\mathrm{MCI}$, their relative lack of sensitivity to detecting subtle cognitive impairment has been well documented (McKhann et al. 2011;

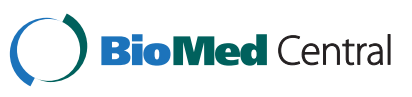


Proust-Lima et al. 2007) as has their potential for idiosyncratic errors in administration (Miller et al. 2008; Miller et al. 2011). Furthermore, although items on these bedside screening instruments are selected to assess a wide variety of cognitive domains, subscale scores on these instruments generally have low validity and reliability (Strauss et al. 2006).

The CogState Brief Battery (CBB) is a brief, computeradministered cognitive test battery that requires approximately 10 minutes for administration and consists of four cognitive tasks that measure psychomotor function, attention, working memory and memory (Darby et al. 2012; Fredrickson et al. 2010; Maruff et al. 2009). The sensitivity of the $\mathrm{CBB}$ to detect cognitive impairment in several neurodegenerative conditions has been demonstrated in prior work (Darby et al. 2009; Hammers et al. 2012; Lim et al. 2012a). Given that the CBB is computerized, the administration, scoring and reporting is automated and highly standardized. Each task in the battery is constructed using playing cards as stimuli with the test taker required to answer only "yes" or "no" on each trial in accord with a simple rule. The simple stimuli, rules and responses have been combined to generate cognitive paradigms that have been well-validated in neuropsychological and cognitive studies. These include measures of psychomotor function (Detection task), visual attention (Identification task), working memory (One Back task) and visual learning set within a pattern separation model (One Card Learning task, (Fredrickson et al. 2010; Maruff et al. 2009)). The simplicity of the CBB has allowed it to be applied successfully to the measurement of cognitive function in healthy older adults and in adults with clinically diagnosed and prodromal AD (Darby et al. 2009; Lim et al. 2012a, b). These studies have found that performance on the CBB working memory and learning tasks are sensitive to cognitive impairment in clinically diagnosed $\mathrm{AD}$ as well as its prodromal stage; amnestic MCI. Furthermore, the CBB was designed specifically for repeated administration, as it can be administered repeatedly without generating significant practice effects (Collie et al. 2003; Falleti et al. 2006), including in healthy older people (Fredrickson et al. 2010). The CBB has been shown to be sensitive to AD-related cognitive decline in healthy older adults and in adults with amnestic MCI (Darby et al. 2002, 2012; Lim et al. 2013a, b) as well as to improvement in cognition arising from treatment with putative cognitive enhancing drugs such as donepezil (Jaeger et al. 2011), histamine H3 antagonists (Nathan et al. 2013) and testosterone (Davison et al. 2011) in older people.

Recent data from studies using the $\mathrm{CBB}$ suggests that composite scores, which are constructed from aggregating performance on the Detection and Identification tasks (i.e., an attention/psychomotor composite) and the learning and working memory tasks (i.e., a learning/ working memory composite) may have greater sensitivity to both AD-related cognitive impairment and decline when compared to scores from the individual CBB tasks (Lim et al. in press, 2012b, c). This increased sensitivity of cognitive composite scores over individual test scores is consistent with current neuropsychological models that emphasise the benefit of composite scores in clinical research (Nuechterlein et al. 2008).

While the $\mathrm{CBB}$ is not intended to replace formal neuropsychological assessment, the results of these recent studies do converge to suggest that it may be useful as a screening test for AD-related cognitive impairment in clinical settings. However, the clinical utility of the $\mathrm{CBB}$ in screening for $\mathrm{AD}$-related cognitive impairment has not been established formally. To achieve this, it is necessary to compute estimates of sensitivity and specificity of each composite score and identify their optimal value for the identification of cognitive impairment related to both $\mathrm{AD}$ and MCI. It is also necessary to understand the nature of any relationship between each composite measure and cognitive impairment across disease severity. Finally, establishing the reliability and stability of these composite scores would facilitate the use of composite cognitive measures to monitor changes in cognitive function in clinical or prodromal AD.

The main aim of this study was to determine the sensitivity, specificity and reliability of the $\mathrm{CBB}$ composite scores for the detection and monitoring of cognitive impairment in aging and dementia (Lim et al. 2012a, b). The first hypothesis was that the attention/psychomotor and learning/working memory composites would be sensitive to AD-related cognitive impairment although the sensitivity of the learning/working memory composite would be greater than that of the attention/psychomotor composite. We then examined the relationship between each cognitive composite score and disease severity across the clinical groups. Our second hypothesis was that on reassessment, both cognitive composite scores would show high test-retest reliability and stability in healthy adults, amnestic $\mathrm{MCI}$ and $\mathrm{AD}$.

\section{Methods}

\section{Participants}

Participants in the current study were recruited from the Australian Imaging, Biomarkers and Lifestyle (AIBL) Study of Ageing (Ellis et al. 2009; Rowe et al. 2010) and from hospital clinics specializing the diagnoses of $\mathrm{AD}$ who had completed the CBB successfully as part of their assessment (Lim et al. 2012a). The process of recruitment and diagnostic classification been described in detail previously for the AIBL (Ellis et al. 2009) and clinical samples (Maruff et al. 2004). Of the AIBL participants who had completed the CBB, 659 healthy adults (HA), 
72 adults who met clinical criteria for amnestic MCI and 51 adults who met clinical criteria for mild to moderate AD (Ellis et al. 2009) were recruited into the study. For the hospital clinical sample 35 patients who met clinical criteria for amnestic MCI were recruited (Maruff et al. 2004). Briefly, all patients underwent a detailed diagnostic workup by clinician specializing in $\mathrm{AD}$ on the basis of clinical, neuropsychological and structural neuroimaging data. All cases of amnestic MCI were classified using established criteria (Petersen et al. 1999; Winblad et al. 2004). All cases of AD met NINCDS-ADRDA criteria for AD (McKhann et al. 1984). To increase the reliability of classification, all individuals classified with MCI and $\mathrm{AD}$ were required to meet the criteria for these clinical classifications on two consecutive assessments. Data from the $\mathrm{CBB}$ was not used by clinicians to classify any individual's clinical status. For participants with AD, additional inclusion criteria included a score of 18 to 26 on the MMSE (Folstein et al. 1975). The severity of dementia was rated in patients with $\mathrm{AD}$ and MCI using the Clinical Dementia Rating (CDR) scale to provide a sum of boxes score and an overall CDR score (Morris 1983). For all participants, exclusion criteria for the study included: schizophrenia; depression (15-item Geriatric Depression Score (GDS) of 6 or greater); Parkinson's disease; cancer (except basal cell skin carcinoma) within the last two years; symptomatic stroke; uncontrolled diabetes; or current regular alcohol use exceeding two standard drinks per day for women or four per day for men. None of the control or MCI group were taking psychotropic drugs or cholinesterase inhibitors although each of the patients with $\mathrm{AD}$ were taking cholinesterase inhibitors. Demographic and clinical characteristics of the HC, MCI and AD groups are shown in Table 1. The study complied with the regulations of three institutional research and ethics committees (Ellis et al. 2009), and all participants gave written informed consent prior to participation in the study. To assess test-retest reliability, we re-assessed $115 \mathrm{HA}, 47$ adults with $\mathrm{MCI}$, and 43 adults with $\mathrm{AD}$ who underwent serial assessments on the computerized cognitive battery. These individuals were assessed monthly over four months (Lim et al. 2013b). The process of recruitment and additional inclusion and exclusion criteria for this subgroup of AIBL participants has been described in detail previously (Lim et al. 2013b).

\section{Measures}

\section{Demographic and clinical characteristics}

Participants underwent a series of comprehensive demographic, health and cognitive tests performed by trained research assistants under the supervision of licensed clinical neuropsychologists. Participants' age was based on self-report, and this information was corroborated by
Table 1 Demographic and clinical characteristics for each clinical group

\begin{tabular}{|c|c|c|c|}
\hline & $\mathrm{HC}(n=659)$ & $\mathrm{MCl}(n=107)$ & $\mathrm{AD}(n=51)$ \\
\hline & $\overline{\text { Mean (SD) }}$ & Mean (SD) & $\overline{\text { Mean (SD) }}$ \\
\hline Percentage females $^{+}$ & $57.8 \%$ & $50.5 \%$ & $51.0 \%$ \\
\hline Age (years) & $69.5(6.6)$ & $75.7(7.5)$ & $79.3(7.2)$ \\
\hline MMSE & $28.7(1.4)$ & $26.1(2.1)$ & $19.8(3.8)$ \\
\hline CDR-SB & $0.06(0.3)$ & $1.39(1.2)$ & $5.87(2.4)$ \\
\hline Premorbid IQ & $108.35(7.3)$ & $105.9(9.0)$ & $103.2(8.4)$ \\
\hline Education level med & $12(9-15)$ & $12(9-15)$ & $12(9-15)$ \\
\hline HADS depression & $2.6(2.2)$ & $3.3(2.4)$ & $3.8(3.1)$ \\
\hline HADS anxiety & $4.3(2.9)$ & $4.1(2.6)$ & $4.7(3.7)$ \\
\hline Detection speed ${ }^{*}$ & $100.0(10.0)$ & $94.26(13.7)$ & $91.72(13.5)$ \\
\hline Identification speed* & $100.0(10.0)$ & $87.62(16.4)$ & $84.12(15.4)$ \\
\hline $\begin{array}{l}\text { One card learning } \\
\text { accuracy* }\end{array}$ & $100.0(10.0)$ & $83.74(11.6)$ & $78.42(15.1)$ \\
\hline One back accuracy* & $100.0(10.0)$ & $79.18(13.1)$ & $70.14(16.3)$ \\
\hline
\end{tabular}

Note: $+=$ percentage of clinical group, med $=$ median (range), ${ }^{*}=$ mean score $=100$ and SD score $=10$ because the mean and SD of the controls was used to standardize the data for each individuals performance on each cognitive task. One way ANOVAs indicated significant differences between groups on age, premorbid $\mathrm{IQ}$, and depressive symptoms, all $p^{\prime} \mathrm{s}<0.001$. MMSE = Mini Mental State Examination; CDR-SB = Clinical Dementia Rating Scale, Sum of Boxes Score; HADS $=$ Hospital Anxiety and Depression Scale.

a family member. Additionally, the MMSE, CDR, Wechsler Test of Adult Reading (WTAR) (Wechsler 2001) and the Hospital Anxiety and Depression Scale (HADS) (Snaith \& Zigmond 1986) were administered to participants to measure overall cognitive impairment, general clinical function, premorbid IQ, and level of anxiety and depressive symptoms, respectively.

\section{CogState brief battery}

The four tasks from the CBB have been described in detail previously (Darby et al. 2012; Lim et al. 2012a, b), and they are summarized here. On each trial of each task, a single playing card stimulus was presented in the centre of the computer screen. The values, color and suit of the playing cards were determined by the requirements of each task. At the presentation of each playing card stimulus, participants were required to respond either "yes" or "no" by pressing a "yes" or "no" button attached to the computer through a USB port. The yes button was always placed on the right and pressed with the right hand and the no button was placed on the left and pressed with the left hand. Patients were instructed to press the "yes" or "no" button as quickly and as accurately as possible. At the beginning of each task, task rules were presented on the computer screen, and also given verbally to the participant by the supervisor. This was followed by an interactive demonstration in which participants practiced the task. Once the practice trials were complete, the task began. The four tasks were 
presented in the same order. For each task, the speed and accuracy of each response to each trial was recorded and expressed as a mean reaction time (in milliseconds) and accuracy (proportion correct). For each task a single performance measure has been selected on the basis that it comes from a normal data distribution, has no floor or ceiling effects, does not have restricted range and has good reliability, stability and sensitivity to change (Fredrickson et al. 2010; Hammers et al. 2011). The tasks from the CBB are described in their order of administration below.

The Detection (DET) task is a simple reaction time test shown to measure psychomotor function. In this task, the participant must attend to the card in the center of the screen and respond to the question "has the card turned over?" Participants were instructed to press the "Yes" button as soon as the card turns face up. The face of the card is always the same generic joker card. The task ends after 35 correct trials have been recorded. Trials on which anticipatory responses occurred were excluded and another trial was given so that all participants completed the 35 trials. The primary performance measure for this task was reaction time in milliseconds (speed), which was normalized using a logarithmic base $10\left(\log _{10}\right)$ transformation.

The Identification (IDN) task is a choice reaction time test shown to measure visual attention. In this task, the participant must attend to the card in the center of the screen, and respond to the question "Is the card red?" Participants were required to press the "Yes" button if it is and the "No" button it is not. The face of the cards displayed were either red or black joker cards in equivalent numbers in random order. These cards were different to the generic joker card used in the DET task. The task ends after 30 correct trials. Trials on which anticipatory responses occurred were excluded and another trial was given so that all participants completed the 30 trials. The primary performance measure for this task was reaction time in milliseconds (speed), which was normalized using a $\log _{10}$ transformation.

The One Card Learning (OCL) task is a continuous visual recognition learning task that assesses visual learning within a pattern separation model (Yassa et al. 2010). Theoretical models of pattern separation model specify that information is organized in orthogonal and distinct nonoverlapping representations so that that new memories can be stored rapidly without interference (Norman \& O'Reilly 2003). In this task the participant must attend to the card in the center of the screen and respond to the question "have you seen this card before in this task?" If the answer was yes, participants were instructed to press the "Yes" button, and the "No" button if the answer was no. Normal playing cards were displayed (without joker cards). In this task, six cards are drawn at random from the deck and are repeated throughout the task. These four cards are interspersed with distractors (non-repeating cards). The task ends after 80 trials, without rescheduling for post-anticipatory correct trials. The primary performance measure for this task was the proportion of correct answers (accuracy), which was normalized using an arcsine square-root transformation.

The One-Back (OBK) task is a task of working memory and attention. Similar in presentation to the OCL task, participants must attend to the card in the center of the screen and respond to the question "is this card the same as that on the immediately previous trial?" If the answer was yes, participants were instructed to press the "Yes" button, and the "No" button if the answer was no. The task ends after 30 correct trials. A correct but post-anticipatory response led to scheduling of an extra trial. The primary performance measure for this task was the proportion of correct answers (accuracy), which was normalized using an arcsine square-root transformation.

\section{Data analysis}

For each participant, each performance measure from the four tasks in the $\mathrm{CBB}$ was computed as reported previously (Lim et al. 2012a). For each performance measure, the mean and standard deviation (SD) was computed for the HA group according to their age in deciles (e.g., 51-60, 61-70, 71-80, 81-90). These means and SDs were then used to standardize scores on each of the four cognitive tasks for each participant. A learning/ working memory composite score was computed by averaging the standardized scores for the OCL and OBK tasks, and an attention/psychomotor function composite score was computed by averaging the standardized scores for the DET and IDN tasks. For each individual, both composite scores were then re-standardized using the mean and SD for each composite score computed from the $\mathrm{HC}$ group and then transformed once more so that each had a mean of 100 and a standard deviation of 10. This was achieved by first multiplying each standardized score by 10 and then adding 100. If data for one or both of the tasks that contributed to each composite was missing, the composite score was not computed. There was no missing data for the attention/psychomotor function composite and 26 ( $\mathrm{HA}=17$ cases, $\mathrm{AD}=9$ cases) missing data for the learning/working memory composite score.

To evaluate the first hypothesis that the composite scores would be sensitive to AD-related cognitive impairment, we conducted two analysis of covariance (ANCOVA), with age, premorbid IQ, and level of depressive symptoms entered as covariates. For each composite score, Hedge's $g$ was used to quantify the magnitude of impairment in each of the clinical groups relative to the healthy controls. We also determined the 
extent to which performance on each composite was worse in the AD group than in the MCI using ANCOVA with age, premorbid IQ, and level of depressive symptoms entered as covariates. Once again for each comparison Hedge's $g$ was used to quantify the magnitude of impairment in the AD group relative to the MCI group. Receiver operating characteristic (ROC) curves were then generated to illustrate the relationship between clinical sensitivity and specificity of each composite for classification of MCI and AD groups, as measured by the area under the curve (AUC) statistic. AUC values were compared to those obtained for the MMSE in the same analyses with statistical significance indicated when $95 \%$ confidence intervals for each estimate did not overlap. For classification of cognitive impairment in MCI and AD, the value of each composite score that provided the optimal balance between sensitivity and specificity was identified from the ROC curve using Youden's J statistic (Swets 1996). The predictive power of the combination of the optimum cut-score for each composite in predicting MCI and AD was then determined by computing the odds ratios for the classification of cognitive impairment in each clinical group (versus the $\mathrm{HC}$ group). Finally the relationship between the cognitive composite scores and disease severity was determined by collapsing data for the MCI and $\mathrm{AD}$ group and classifying each individual according to their score on the CDR Sum of Boxes score. Curve fitting analysis was then used to determine the extent to which scores on each of the cognitive composites was associated with increased CDR Sum of Boxes scores.

To evaluate our second hypothesis that the cognitive composite scores would show high test-retest reliability and stability, we computed mean change scores and test-retest reliability statistics over four months for the two CogState composite scores. This was conducted in a subgroup of AIBL participants who had consented to serial computerized cognitive assessments (Lim et al. 2013b). Average measure intraclass correlation coefficients (ICC) were used to compute the test-retest reliability of the two composites, in both the total group and in each clinical classification group separately.

\section{Results}

\section{Cognitive function in healthy controls}

In the HA group, the attention/psychomotor composite was not associated significantly with premorbid IQ $(r=0.07, p>0.05)$ or level of education. It was associated significantly with levels of depressive $(r=0.11, p<0.05)$ and anxiety symptoms $(r=0.10, p<0.05)$. The learning/ working memory composite was not associated significantly with premorbid IQ $(r=-0.06, \mathrm{p}>0.05)$, or levels of depressive $(r=0.02, \mathrm{p}>0.05)$, or anxiety symptoms $(r=0.01, \mathrm{p}>0.05)$.

\section{Magnitude of cognitive impairment in $\mathrm{MCl}$ and $\mathrm{AD}$}

As has been reported previously (Lim et al. 2012a), comparison of the demographic variables between clinical groups indicated significant differences in age, premorbid IQ, and level of depressive symptoms (see Table 1). As such, these variables were included as covariates in comparisons of the $\mathrm{CBB}$ composite measures between groups.

Results of the ANCOVAs revealed statistically significant group differences for the learning/working memory composite, $F(2,769)=305.56, p<0.001$, and the attention/psychomotor function composite, $F(2,794)=26.52$, $p<0.001$. Post-hoc comparisons indicated that adults with $\mathrm{MCI}$ and $\mathrm{AD}$ performed significantly worse than $\mathrm{HC}$ on the learning/working memory composite, and the magnitudes of these differences were, by convention, large (MCI $g=2.15,95 \% \mathrm{CI}=1.91,2.38$; AD $g=3.18$, $95 \% \mathrm{CI}=2.91,3.28)$. The AD group also performed significantly worse than the MCI group on the learning/ working memory score with this difference moderate in magnitude $(g=0.8495 \% \mathrm{CI}=0.49,1.18 ; \mathrm{p}<0.01)$. Adults with $\mathrm{MCI}$ and $\mathrm{AD}$ also performed significantly worse than HA on the attention/psychomotor composite, although these differences were moderate-to-large in magnitude (MCI $g=0.51,95 \% \mathrm{CI}=0.30,0.72$; $\mathrm{AD} g=1.03$, $95 \% \mathrm{CI}=0.73,1.33)$. The $\mathrm{AD}$ group also performed significantly worse than the MCI group on the attention/ psychomotor function score with the differences moderate in magnitude (is $g=0.4095 \% \mathrm{CI}=0.07,0.74$ ).

\section{Sensitivity and specificity of CBB composite scores in assessing cognitive impairment in $\mathrm{MCl}$ and $\mathrm{AD}$}

Inspection of the AUC statistics from the ROC analyses indicated that, by convention, the ROC curves for the learning/working memory composite showed excellent classification accuracy in both MCI and AD ((Swets 1996); Table 2; Figure 1). Accuracy of classification of both MCI and AD was lower for the attention/psychomotor composite (see Table 2, Figure 1). AUC values for the learning/working memory composite were significantly larger (i.e. no overlap between 95\% CIs for AUC values) than for those for the attention/psychomotor composite and for classifying cognitive impairment in both MCI and AD (Table 2). Using the same criteria, the AUC for the learning/working memory composite was also significantly greater than the AUC for MMSE for classifying cognitive impairment in MCI (Table 2). Inspection of the Youden J statistics for the ROC curve for the learning/working memory composite indicated that the cut score that had optimal sensitivity and specificity in classifying cognitive impairment in MCI was 90 (i.e., $\mathrm{z}<=-1 \mathrm{SD}$ ). Application of this same cut score to classification of cognitive impairment in AD yielded a sensitivity of $100 \%$ at the same specificity (Table 2 ). 
Table 2 Areas under ROC curves for $\mathrm{MCl}$ and AD groups relative to healthy controls

\begin{tabular}{|c|c|c|c|c|c|c|}
\hline Composite & $\begin{array}{l}\text { Clinical } \\
\text { group }\end{array}$ & $\begin{array}{c}\text { Sensitivity }(95 \% \mathrm{Cl}) \\
\text { score }<90\end{array}$ & $\begin{array}{c}\text { Specificity }(95 \% \mathrm{Cl}) \\
\text { score }<90\end{array}$ & $\begin{array}{l}\text { Area under ROC } \\
\text { curve }(95 \% \mathrm{Cls})\end{array}$ & Standard error & $p$ \\
\hline \multirow[t]{3}{*}{ Psychomotor/attention } & $A D$ & $52.9 \%(38.5 \%, 67.1 \%)$ & $85.7 \%(82.8 \%, 88.3 \%)$ & $0.73(0.64,0.82)$ & 0.05 & $<0.0001$ \\
\hline & $\mathrm{MCl}$ & $41.1 \%(31.7 \%, 51.1 \%)$ & $85.7 \%(82.8 \%, 88.3 \%)$ & $0.67(0.61,0.73)$ & 0.03 & $<0.0001$ \\
\hline & & Score $<90$ & Score $<90$ & & & \\
\hline \multirow[t]{2}{*}{ Learning/working memory } & $A D$ & $100.0 \%(91.5 \%, 100.0 \%)$ & $84.7 \%(81.7 \%, 87.4 \%)$ & $0.99(0.98,1.00)$ & 0.01 & $<0.0001$ \\
\hline & $\mathrm{MCl}$ & $80.4 \%(71.6 \%, 87.4 \%)$ & $84.7 \%(81.7 \%, 87.4 \%)$ & $0.91(0.87,0.94)$ & 0.02 & $<0.0001$ \\
\hline
\end{tabular}

Note: $\mathrm{ROC}=$ receiver operating characteristic; $\mathrm{MCl}=$ mild cognitive impairment; $\mathrm{AD}=$ Alzheimer's disease; Attention/psychomotor composite $=$ average of the standardized Detection and Identification scores; Learning/working memory composite = average of the standardized One Card Learning and One Back scores; MMSE $=$ Mini Mental State Examination .

\section{Prediction of $\mathrm{MCl}$ and $\mathrm{AD}$ from combined composite scores}

Table 3 shows the odds ratios for classification of MCI or $\mathrm{AD}$ (versus $\mathrm{HA}$ ) for the combination of cognitive impairment of a score $\leq 90$ on the learning/working memory composite and $>/=90$ on the attention/psychomotor composite. This analysis showed that with these cut scores, individuals were 26 times more likely to meet clinical criteria for $\mathrm{MCI}$, and 30 times more likely to meet clinical criteria for AD.

\section{Relationship to disease severity}

For the relationship between MMSE scores and the attention/psychomotor composite, trend analysis indicated no statistically significant relationships in any clinical group. The relationship between MMSE scores and the learning/working memory composite was best described by a linear function in both the MCI $(r=0.38)$ and AD $(r=0.12)$ groups, although this relationship was statistically significant only for the MCI group.

For the relationship between CDR sum of boxes scores and the attention/psychomotor composite, trend analysis indicated that when both $\mathrm{MCI}$ and $\mathrm{AD}$ groups were collapsed, there was a statistically significant linear relationship between increasing disease severity and worse performance on the attention/psychomotor composite (Figure 2a). Similarly, statistically significant linear relationships were observed between CDR sum of boxes scores and the learning/working memory composite when both the MCI and AD groups were collapsed (Figure $2 \mathrm{~b}$ ).

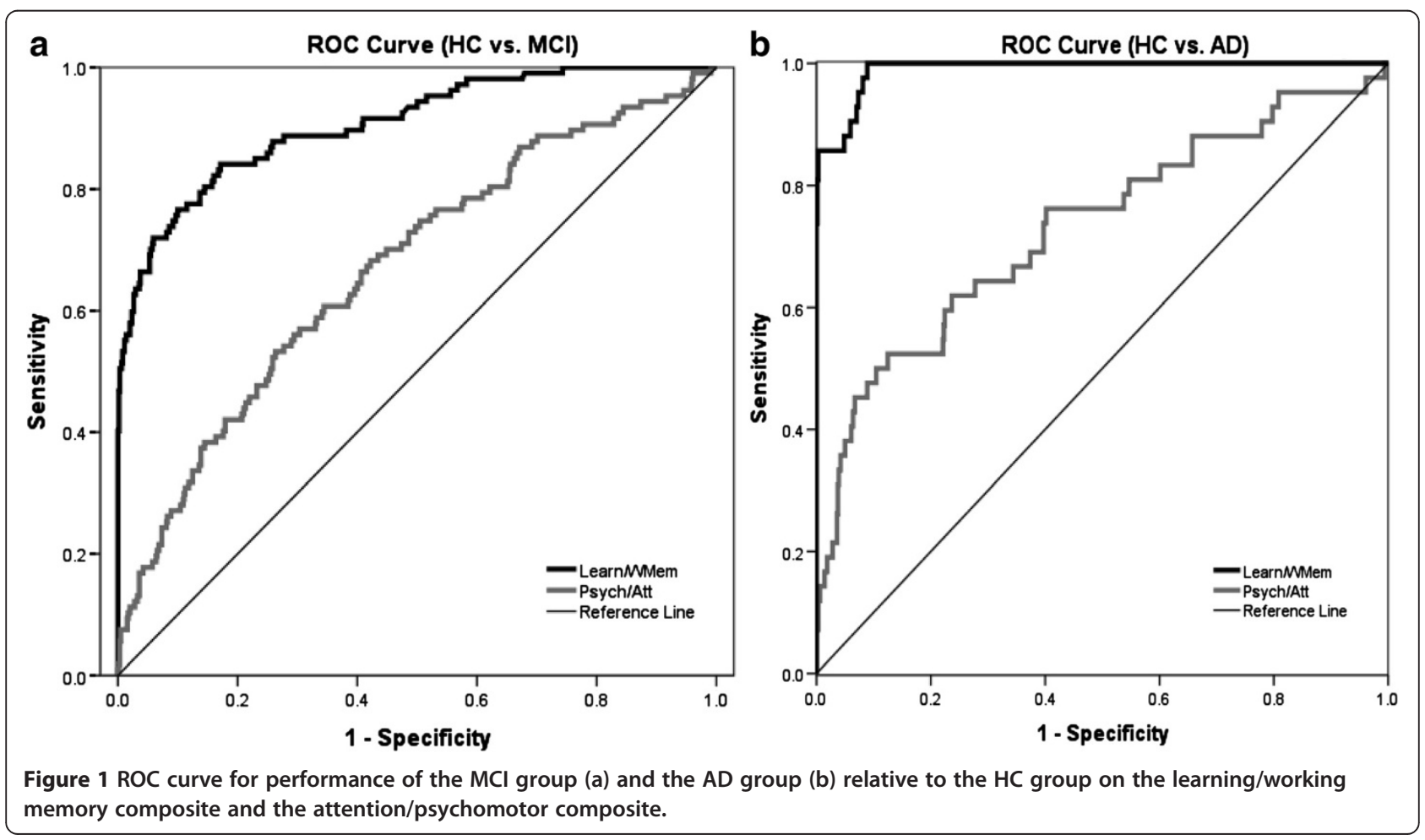


Table 3 Odds ratio, with impaired memory defined as scores of $<90$

\begin{tabular}{|c|c|c|c|c|c|}
\hline & $\begin{array}{l}\text { Normal memory normal } \\
\text { attentional function }(\mathrm{N})\end{array}$ & $\begin{array}{l}\text { Impaired memory normal } \\
\text { attentional function }(\mathrm{N})\end{array}$ & & $\begin{array}{l}\text { Odds ratio (accuracy } \\
\text { impaired) }\end{array}$ & $p$ \\
\hline Healthy controls $(\mathrm{HC})$ & 480 & 84 & & & \\
\hline Mild cognitive impairment (MCl) & 12 & 55 & $\mathrm{HC}$ vs. $\mathrm{MCl}$ & $26.19(13.45,50.98)$ & $<0.0001$ \\
\hline Alzheimer's disease (AD) & 4 & 21 & $H C$ vs. $A D$ & $30.00(10.05,89.60)$ & $<0.0001$ \\
\hline
\end{tabular}

\section{Test-retest reliability}

The ICC for both composites are shown in Table 4. When considered according to clinical classification, both composites demonstrated high (i.e., $r>0.70$ ) testretest reliability over a four month assessment period and these estimates were equivalent between the clinical groups (see Table 4).

\section{Discussion}

Results of this study supported our first hypothesis that the learning/working memory composite and the attention/psychomotor composite, derived from the outcome measures on the $\mathrm{CBB}$, would be sensitive to detecting cognitive impairment in $\mathrm{MCI}$ and $\mathrm{AD}$. In $\mathrm{AD}$, we observed a large impairment for both cognitive composite scores, although the magnitude of impairment on the learning/ working memory composite was much greater than that for the attention/psychomotor composite. Neuropsychological models of the cognitive tasks that contribute to the learning/working memory composite suggest that normal performance on these tasks is likely to depend on the integrity of the hippocampus and temporal lobe (i.e. pattern separation, e.g., Yassa et al. 2010) and prefrontal cortex and anterior cingulate (i.e. working memory, Andrewes 2001; Lezak 1995). Normal performance on the tasks that contribute to attentional functions are likely to depend on integrity of subcortical brain regions including the basal ganglia as well as cortical regions such as the prefrontal and parietal cortices (Andrewes 2001; Lezak 1995). The presence of a relatively greater impairment in cognitive functions dependent on cortical and limbic brain regions (i.e., learning and working memory) with relatively subtle impairment in motor and attentional functions is consistent with neuropsychological models of AD which emphasise that cognitive impairment characteristic of both prodromal and clinically classified $\mathrm{AD}$ is disruption to memory and executive function (Baddeley et al. 1991; Kensinger et al. 2003; McKhann et al. 2011). This pattern of impairment is also consistent with the predilection of AD-related neuronal loss in the medial temporal lobe and other cortical brain areas (Jack et al. 2009; Villemagne et al. 2013).

Differences in the nature of impairment for the two cognitive composite scores were also evident in their
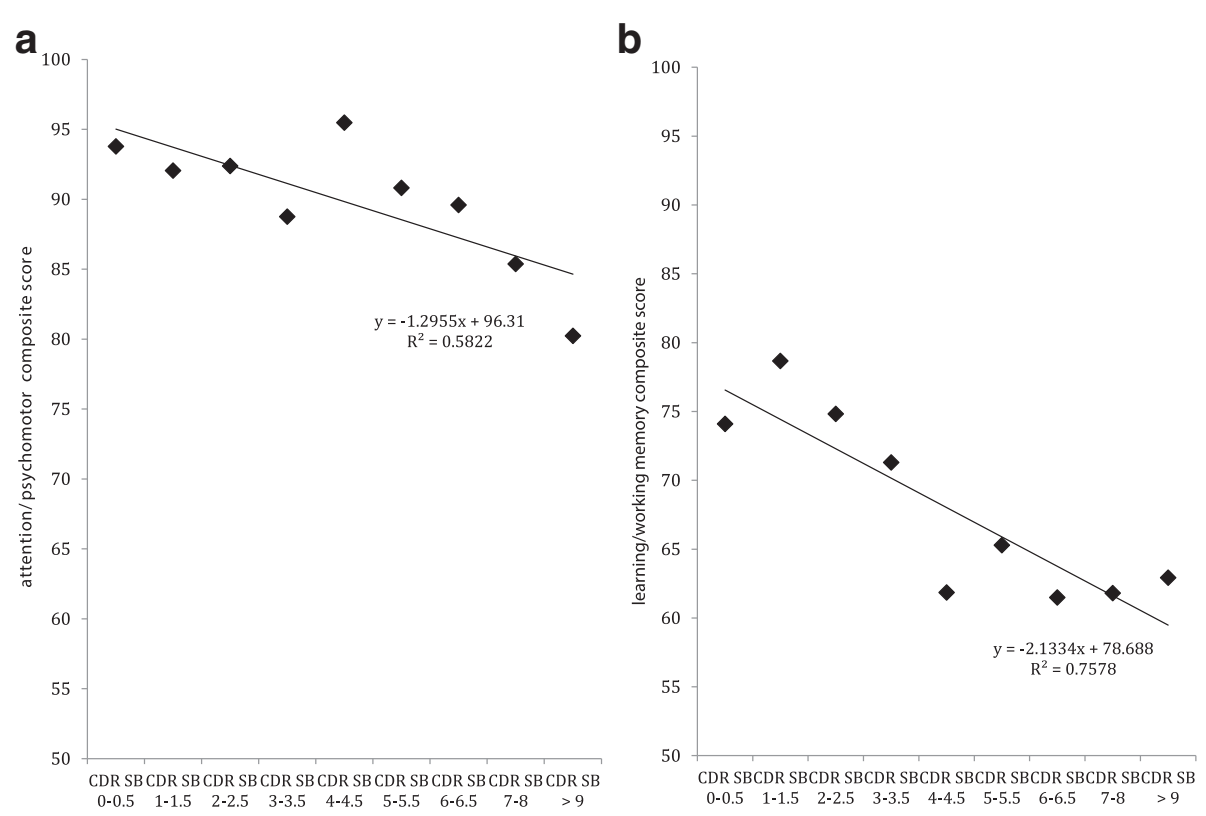

Figure 2 Relationship between performance on the CDR Sum of Boxes and the attention/psychomotor composite (a) and the learning/ working memory composite (b) in individuals with $\mathbf{M C l}$ and $\mathbf{A D}$. The diamond markers on each figure represent the mean composite score for each group of individuals with the same score on the CDR-SOB. 
Table 4 Test-retest reliability and group mean (standard deviation) of each clinical group over a four month assessment period

\begin{tabular}{|c|c|c|c|c|c|c|c|c|c|c|c|}
\hline & \multirow[b]{2}{*}{ Composite } & \multirow[b]{2}{*}{ ICC (95\% CI) } & \multirow[b]{2}{*}{$p$} & \multicolumn{2}{|c|}{ Month 1} & \multicolumn{2}{|c|}{ Month 2} & \multicolumn{2}{|c|}{ Month 3} & \multicolumn{2}{|c|}{ Month 4} \\
\hline & & & & Mean & SD & Mean & SD & Mean & SD & Mean & SD \\
\hline \multirow[t]{2}{*}{ Overall } & Attention & $0.90(0.87,0.92)$ & $<.0001$ & 96.46 & 11.97 & 95.79 & 15.65 & 95.66 & 11.79 & 97.37 & 23.16 \\
\hline & Memory & $0.95(0.93,0.96)$ & $<.0001$ & 93.03 & 14.09 & 94.16 & 13.3 & 95.17 & 14.6 & 96.39 & 14.76 \\
\hline \multirow[t]{2}{*}{$\mathrm{HC}$} & Attention & $0.94(0.92,0.96)$ & $<.0001$ & 100 & 8.86 & 98.86 & 9.08 & 99.26 & 8.81 & 101.27 & 27.59 \\
\hline & Memory & $0.78(0.70,0.85)$ & $<.0001$ & 99.92 & 8.05 & 100.73 & 8.37 & 102.61 & 8.86 & 104.57 & 7.39 \\
\hline \multirow[t]{2}{*}{$\mathrm{MCl}$} & Attention & $0.94(0.90,0.97)$ & $<.0001$ & 95.69 & 11.73 & 93.66 & 11.6 & 93.9 & 12.53 & 95.42 & 11.33 \\
\hline & Memory & $0.86(0.78,0.92)$ & $<.0001$ & 92.18 & 10.99 & 92.16 & 9.51 & 92.53 & 11.03 & 93.89 & 10.02 \\
\hline \multirow[t]{2}{*}{$A D$} & Attention & $0.77(0.58,0.89)$ & $<.0001$ & 86.76 & 14.83 & 88.96 & 28.53 & 86.56 & 13.8 & 88.04 & 14.65 \\
\hline & Memory & $0.91(0.84,0.96)$ & $<.0001$ & 73.4 & 13.07 & 75.95 & 12.26 & 75.1 & 13.11 & 75.16 & 13.46 \\
\hline
\end{tabular}

Note: ICC = Intra-class correlation coefficient; $\mathrm{HC}=$ healthy controls; $\mathrm{MCl}=$ mild cognitive impairment; $\mathrm{AD}=$ Alzheimer's disease; Attention/psychomotor composite = average of the standardized Detection and Identification scores; Learning/working memory composite $=$ average of the standardized One Card Learning and One Back scores.

sensitivity to detecting AD-related cognitive impairment in individuals. The learning/working memory composite was most sensitive to AD-related cognitive impairment with $100 \%$ of $\mathrm{AD}$ cases classified as impaired when the criterion for abnormality was set at a score of 90 . When the criterion for abnormality was decreased to 80 , the sensitivity for abnormality decreased to only $86 \%$ (Figure 1 ). As expected, the attention/psychomotor composite showed lower levels of sensitivity, with only $53 \%$ of $\mathrm{AD}$ cases identified when sensitivity was set at the least conservative level (i.e., score of 90). Taken together, these data indicate that with the use of these composite scores, cognitive impairment in AD will present as a relatively large impairment in working memory and learning and with relatively intact psychomotor and attentional functions. The nature and magnitude of this cognitive impairment is consistent with the descriptions of $\mathrm{AD}$ cases from the neuropsychological literature (Andrewes 2001; McKhann et al. 2011). While it is unsurprising that patients with clinical defined $\mathrm{AD}$ showed poor performance on a measure of learning and working memory, the high specificity of the learning/working memory composite, with the lesser impairment on the attention/psychomotor composite also indicates that the CogState tests themselves can be used effectively in patients with $\mathrm{AD}$ and suggests further that this pattern of performance may even be useful to clinicians investigating the aetiology of cognitive impairment in older adults.

As expected, in adults with MCI, cognitive impairment was qualitatively similar but quantitatively less pronounced to that observed for clinically diagnosed AD. Compared to healthy adults, the MCI group showed large impairment on the learning/working memory composite $(g=2.2)$, although not as great as that observed for the same composite in $\mathrm{AD}$. While performance on the attention/psychomotor composite was also impaired compared to healthy adults, the magnitude of this impairment was only moderate $(g=0.51)$. Once again this impairment was less than that observed for the same composite in AD. Despite these impairment, performance on both the attention/psychomotor function and learning working memory composites in the MCI group was superior to that in the AD group. When considered for individuals, a score of $\leq 90$ on the learning/working memory composite had optimal sensitivity and specificity for detecting cognitive impairment in MCI. At the optimum cut score for the attention/psychomotor composite, the sensitivity was only $40 \%$, with a specificity of $85 \%$. Therefore, as was observed for AD, cognitive impairment in $\mathrm{MCI}$ was characterised best as a large abnormality in working memory and learning with relatively normal psychomotor and attentional function. The likelihood that a combination of abnormal performance on the learning/working memory composite with normal performance on the attention/psychomotor composite could predict $\mathrm{MCI}$ or $\mathrm{AD}$ was very high, since individuals who met this criteria were 26 times more likely to have MCI or 30 times more likely to have AD than those who did not meet the criteria.

For the relationship between cognition and disease severity in the $\mathrm{MCI}$ and $\mathrm{AD}$ groups, while a significant linear relationship was observed between disease severity and the attention/psychomotor composite, this relationship was driven mainly by individuals with the most extreme scores on the severity measure. Furthermore the magnitude of this relationship was only small. In contrast to these more reflexive aspects of cognition, disease severity was strongly associated with the learning/working memory composite.

The second hypothesis that the attention/psychomotor composite and the learning/working memory composite would show high test-retest reliability and stability in healthy adults, adults with MCI and AD, was also supported. Assessments on the same tests conducted four times in three months showed that both composite scores remained stable and showed test-retest reliability 
with repeated administration. Thus, despite repeated testing over relatively short retest intervals, including in patients with cognitive impairment, both composites showed no evidence of practice effects, and estimates of within subject variability remained low. Further, estimates of test-retest reliability for each composite were, by convention, high $(r>0.70)$. These results are consistent with findings from earlier clinical studies of MCI and $\mathrm{AD}$ groups, which have shown that performance on the individual tests from the $\mathrm{CBB}$ show little to no practice effects, have high test-retest reliability, and have low within-subject variability (Darby et al. 2012; Fredrickson et al. 2010; Lim et al. 2013b). While individual measures from the CogState battery have been shown to be sensitive to cognitive decline in MCI (e.g. Lim et al. 2012a, b), it will be important now to determine the extent to which composite scores derived in this study will be also sensitive to cognitive decline in MCI.

Taken together, results of this study converge to suggest that the performance on the learning/working memory and attention/psychomotor composites of the CBB can be used to identify reliably cognitive impairment in people with, and at risk of AD. Thus the two composite scores from the $\mathrm{CBB}$ should be useful in screening for cognitive impairment in MCI or AD. The estimates of sensitivity for the composite scores from the $\mathrm{CBB}$ reported here are equivalent or slightly better than those reported previously for other screening instruments used commonly in the early identification of aMCI and AD. For example, estimates of the sensitivity for the MoCA show that the total score has a high sensitivity to $\mathrm{AD}$, while retaining a high specificity. However, as was observed in the current study, the sensitivity of the MoCA to aMCI is also relatively high (81\%; (Freitas et al. 2013)), provided that estimates of lower levels of specificity (e.g. 77\%) are tolerated. As with the MoCA, performance on the MMSE also shows relatively high sensitivity and specificity for identifying cognitive impairment in AD (Freitas et al. 2013; Strauss et al. 2006) although its sensitivity to cognitive impairment in $\mathrm{MCI}$ is lower than the MoCA and that reported here, even if a low specificity is allowed. The equivalence of these estimates occurs mainly because all studies use the same method, where the test instrument is applied to identify cognitive impairment in a group of individuals that has been carefully assessed and undergone relatively rigorous inclusion and exclusion criteria. One strength of the composite scores, observed in this study, was that they were not associated with estimates of premorbid intelligence or depressive symptoms. The psychomotor attention composite was associated with levels of anxiety symptoms although the magnitude of this association was very small. Taken together this analysis of associations suggests that the composite cognitive scores may be useful in settings where issues such as low premorbid intelligence or mood obscure the assessment of cognitive function in individuals undergoing clinical workup for $\mathrm{MCI}$ or $\mathrm{AD}$.

When cognitive assessments are conducted in unselected populations, such as in epidemiological studies, neuropsychological tests are always preferred to bedside screening instruments for the identification of cognitive impairment (Clarke et al. 2000; Ellis et al. 2009; Petersen et al. 2010). This is because neuropsychological tests provide more reliable estimates of individual cognitive functions. Acceptable estimates of validity and reliability are found for bedside screening instruments only when their total score is used, and accordingly, scores of their subscales have been shown to have limited use for describing the nature of cognitive impairment in individuals (Strauss et al. 2006). A limitation of bedside screening instruments for tracking cognitive function is reflected in their absence as outcome measures in clinical trials of drugs designed to improve cognitive function in MCI or $\mathrm{AD}$. This is due to restriction in the range of possible scores for people with dementia; the presence of ceiling effects in data distributions; and the substantial practice effects that occur with repeated administrations. As with other neuropsychological tests, the tasks from the $\mathrm{CBB}$ have been used extensively in epidemiological studies, as well as in clinical trials (Bateman et al. 2011; Ellis et al. 2009). Furthermore associations between performance on the $\mathrm{CBB}$ tasks and that on conventional neuropsychological measures indicate that each task has sound construct validity (Maruff et al. 2009). The data shown here extend these findings to suggest that the two cognitive composite scores that arise from individual measures that comprise the CBB could be applied effectively as a cognitive screening instrument not only for assessing cognitive impairment in dementia, but also in other neurological and psychiatric conditions.

There are some limitations in the current study that warrant consideration in interpreting the results. First, as has been considered already the current data for this study were drawn from studies of $\mathrm{MCI}$ and $\mathrm{AD}$, therefore the high sensitivity and specificity demonstrated here should be challenged in individuals from a clinical setting. Second, while the MCI group recruited here met clinical criteria shown to increase the risk of AD (Petersen et al. 1999), amyloid biomarkers (e.g., Petersen et al. 2010) were not measured in the current analysis. Therefore, although the current data show that the learning/working memory composite score was sensitive to the cognitive impairment that characterizes MCI more study is needed to determine the relationship the relationship between the CogState composite scores and amyloid biomarkers within this clinical classification. These issues notwithstanding the current results do show that the composite scores from the CogState Brief Battery have good potential for use in screening for cognitive impairment related to $\mathrm{MCI}$ and $\mathrm{AD}$. 


\section{Competing interests}

PM is a full-time employee of CogState Ltd. YYL, KE, and CM report no disclosures. DD is a scientific consultant to CogState Ltd. DA has served on scientific advisory boards for Novartis, Eli Lilly, Janssen, and Pfizer Inc.; has received funding for travel from Janssen and Pfizer Inc., has served as Editor-in-Chief for International Psychogeriatrics; has received speaker honoraria from Pfizer Inc. and Lundbeck Inc.; and has received research support from Eli Lilly and Company, GlaxoSmithKline, Forest Laboratories Inc., Novartis, and CSIRO. CS has been partially supported by research fellowships funded by Alzheimer's Australia and the NHMRC. Alzheimer's Australia (Victoria and Western Australia) assisted with promotion of the study and the screening of telephone calls from volunteers. Funding for the study was provided in part by the study partners [Australian Commonwealth Scientific Industrial and research Organization (CSIRO), Edith Cowan University (ECU), Mental Health Research institute (MHRI), Alzheimer's Australia (AA), National Ageing Research Institute (NARI), Austin Health, CogState Ltd., Hollywood Private Hospital, Sir Charles Gardner Hospital, and Astra Zeneca. The study also received support from the National Health and Medical Research Council (NHMRC) and the Dementia Collaborative Research Centres program (DCRC2).

\section{Authors' contributions}

PM, YYL, AS, DD, PHP participated in the design, acquisition and interpretation of the data, and the writing of this manuscript. DA, CS and CLM participated in the study concept and design. All authors contributed to analysis and interpretation of data. PM, AS, YYL participated in the drafting of the manuscript. PM, YYL, DD, KAE, PJS, RHP, DA, AS, CS, AB and CM participated in the critical revision of the manuscript. PM, YYL, AS and RHP participated in the statistical analysis. PM, DA, and KE supervised the study. All authors read and approved the final manuscript.

\section{Author details}

${ }^{1}$ The Florey Institute of Neuroscience and Mental Health, University of Melbourne, Parkville, Victoria, Australia. ${ }^{2}$ CogState Ltd., Melbourne, Victoria, Australia. ${ }^{3}$ Academic Unit for Psychiatry of Old Age, Department of Psychiatry, The University of Melbourne, Kew, Victoria, Australia. ${ }^{4}$ National Ageing Research Institute, Parkville, Victoria, Australia. ${ }^{5}$ Department of Psychiatry, Yale University School of Medicine, New Haven, CT, USA. ${ }^{6}$ Lifespan Hospital System \& Department of Neurology, Warren Alpert Medical School of Brown University, Providence, RI, USA. ${ }^{7}$ CSIRO Preventative Health Flagship, Parkville, Victoria, Australia.

\section{Received: 5 July 2013 Accepted: 16 December 2013}

\section{Published: 23 December 2013}

\section{References}

Albert, MS, DeKosky, ST, Dickson, D, Dubois, B, Feldman, HH, Fox, NC, \& Phelps, $\mathrm{CH}$. (2011). The diagnosis of mild cognitive impairment due to Alzheimer's disease: recommendations from the National Institute on Aging and Alzheimer's Association workgroup. Alzheimer's \& Dementia, 7(3), 270-279.

Andrewes, D. (2001). Neuropsychology: From theory to practice. East Sussex: Psychology Press.

Baddeley, AD, Bressi, S, Della Sala, S, Logie, R, \& Spinnler, H. (1991). The decline of working memory in Alzheimer's disease: a longitudinal study. Brain, 114(6), 2521-2542.

Bateman, RJ, Aisen, PS, De Strooper, B, Fox, NC, Lemere, CA, Ringman, JM, \& Xiong, C. (2011). Autosomal-dominant Alzheimer's disease: a review and proposal for the prevention of Alzheimer's disease. Alzheimer's Research \& Therapy, 3(1), 1-13.

Clarke, PJ, Marshall, WW, Ryff, CD, \& Rosenthal, CJ. (2000). Well-being in Canadian seniors: findings from the Canadian Study of Health and Aging. Canadian Journal on Aging, 19, 139-159.

Collie, A, Maruff, P, Darby, DG, \& McStephen, M. (2003). The effects of practice on the cognitive test performance of neurologically normal individuals assessed at brief test-retest intervals. Journal of the International Neuropsychological Society, 9, 419-428.

Darby, D, Fredrickson, J, Fredrickson, A, Moore, L, Sach, J, \& Woodward, M. (2009). Community screening for early signs of dementia. Alzheimer's \& Dementia, 5(5), 3-4.

Darby, D, Maruff, P, Collie, A, \& McStephen, M. (2002). Mild cognitive impairment can be detected by multiple assessments in a single day. Neurology, 59(7), 1042-1046.
Darby, D, Pietrzak, RH, Fredrickson, J, Woodward, M, Moore, L, Fredrickson, A, \& Maruff, P. (2012). Intra-individual cognitive decline using a brief computerized cognitive screening test. Alzheimer's \& Dementia, 8, 95-104.

Davison, SL, Bell, RJ, Gavrilescu, M, Searle, K, Maruff, P, Gogos, A, \& Davis, SR. (2011). Testosterone improves verbal learning and memory in postmenopausal women: results from a pilot study. Maturitas, 70, 307-311.

Ellis, KA, Bush, Al, Darby, D, De Fazio, D, Foster, J, Hudson, P, \& Group, T. A. R. (2009). The Australian Imaging, Biomarkers and Lifestyle (AIBL) study of aging: Methodology and baseline characteristics of 1112 individuals recruited for a longitudinal study of Alzheimer's disease. International Psychogeriatrics, 21(4), 672-687.

Falleti, MG, Maruff, P, Collie, A, \& Darby, DG. (2006). Practice effects associated with the repeated assessment of cognitive function using the CogState battery at 10-minute, one week, and one month test-retest intervals. Journal of Clinical and Experimental Neuropsychology, 28(7), 1096-1112.

Folstein, MF, Folstein, SE, \& McHugh, PR. (1975). "Mini-mental state": a practical method for grading cognitive state of patients for the clinician. Journal of Psychiatric Research, 12, 189-198.

Fredrickson, J, Maruff, P, Woodward, M, Moore, L, Fredrickson, A, Sach, J, \& Darby, D. (2010). Evaluation of the usability of a brief computerized cognitive screening test in older people for epidemiological studies. Neuroepidemiology, 34, 65-75.

Freitas, S, Simões, MR, Alves, L, \& Santana, I. (2013). Montreal cognitive assessment: validation study for mild cognitive impairment and Alzheimer disease. Alzheimer Disease \& Associated Disorders, 27(1), 37-43.

Hammers, D, Spurgeon, E, Ryan, K, Persad, C, Barbas, N, Heidebrink, J, \& Giordani, B. (2012). Validity of a brief computerized cognitive screening test in dementia. Journal of Geriatric Psychiatry and Neurology, 25(2), 89-99.

Hammers, D, Spurgeon, E, Ryan, K, Persad, C, Heidebrink, J, Barbas, N, \& Giordani, B. (2011). Reliability of repeated cognitive assessment of dementia using a brief computerized battery. American Journal of Alzheimer's Disease \& Other Dementias, 26(4), 326-333.

Jack, CR, Lowe, VJ, Weigand, SD, Wiste, HJ, Senjem, ML, Knopman, DS, \& Initiative, T. A. s. D. N. (2009). Serial PIB and MRI in normal, mild cognitive impairment and Alzheimer's disease: implications for sequence of pathological events in Alzheimer's disease. Brain, 132, 1355-1365.

Jaeger, J, Hårdemark, HG, Zettergren, A, Sjögren, N, \& Hannesdottir, K. (2011). Does repeated daily testing improve measurement sensitivity to the cognitive effects of donepezil in mild-to-moderate Alzheimer's Disease? (Paper presented at the Alzheimer's Association International Conference, Paris, France).

Kensinger, EA, Shearer, DK, Locascio, JJ, Growdon, JH, \& Corkin, S. (2003). Working memory in Alzheimer's disease and early Parkinson's disease. Neuropsychology, 17(2), 230-239.

Lezak, MD. (1995). Neuropsychological Assessment (3rd ed.). New York: Oxford University Press.

Lim, YY, Ellis, KA, Ames, D, Darby, D, Harrington, K, Martins, RN, \& AIBL Research Group. (2013a). A $\beta$ amyloid, cognition and APOE genotype in the preclinical stages of Alzheimer's disease. Alzheimer's \& Dementia, 9(5), 538-45.

Lim, YY, Ellis, KA, Harrington, K, Ames, D, Martins, RN, Masters, CL, \& AIBL, RG. (2012a). Use of the CogState Brief Battery in the assessment of Alzheimer's disease related cognitive impairment in the Australian Imaging, Biomarker and Lifestyle (AIBL) study. Journal of Clinical and Experimental Neuropsychology, 34(4), 345-358.

Lim, YY, Ellis, KA, Harrington, K, Pietrzak, RH, Gale, J, Ames, D, \& AIBL, RG. (2013b). Cognitive decline in adults with mild cognitive impairment and high $A \beta$ amyloid: prodromal Alzheimer's disease? Journal of Alzheimer's Disease, 33(4), 1167-1176.

Lim, YY, Ellis, KA, Pietrzak, RH, Ames, D, Darby, D, Harrington, K, \& AIBL, RG. (2012b). Stronger effect of amyloid load than APOE genotype on cognitive decline in healthy older adults. Neurology, 79, 1645-1652.

Lim, YY, Jaeger, J, Harrington, K, Ashwood, T, Ellis, KA, Stöffler, A, \& Maruff, P. (2013c). Three-month stability of the CogState Brief Battery in healthy older adults, mild cognitive impairment, and Alzheimer's disease: results from the Australian Imaging, Biomarkers, and Lifestyle-Rate of Change Substudy (AIBLROCS). Archives of Clinical Neuropsychology, 28(4), 320-30.

Lim, YY, Pietrzak, RH, Ellis, KA, Jaeger, J, Harrington, K, Ashwood, T, \& Maruff, P. (2013d). Rapid decline in episodic memory in healthy older adults with high amyloid- $\beta$. Journal of Alzheimer's Disease, 33(3), 675-679. doi:10.3233/JAD2012-121516.

Maruff, P, Collie, A, Darby, D, Weaver-Cargin, J, Masters, C, \& Currie, J. (2004). Subtle memory decline over 12 months in mild cognitive impairment. Dement Geriatr Cognitve and Geriatric Disorders, 18(3-4), 342-348. 
Maruff, P, Thomas, E, Cysique, L, Brew, B, Collie, A, Snyder, P, \& Pietrzak, RH. (2009). Validity of the CogState brief battery: relationship to standardized tests and sensitivity to cognitive impairment in mild traumatic brain injury, schizophrenia, and AIDS dementia complex. Archives of Clinical Neuropsychology, 24(2), 165-178.

McKhann, GM, Drachman, D, Folstein, M, Katzman, R, Price, D, \& Stadlan, EM. (1984). Clinical diagnosis of Alzheimer's disease: report of the NINCDSADRDA wok group under the auspices of Department of Health and Human Services Task Force on Alzheimer's disease. Neurology, 34, 939-944.

McKhann, GM, Knopman, DS, Chertkow, H, Hyman, BT, Jack, CRJ, Kawas, CH, \& Phelps, CH. (2011). The diagnosis of dementia due to Alzheimer's disease: recommendations from the National Institute of Aging and the Alzheimer's Association workgroup. Alzheimer's \& Dementia, 7(3), 263-269.

Miller, DS, Hurt, SW, Dewerth-Pallmeyer, A, Samuelson, P, van der Putten, D, Jones, L, \& Butler, A. (2008). Ratings surveillance to identify rater errors (Paper presented at the International Society for CNS Clinical Trials and Methodology, Toronto, Canada).

Miller, DS, Young, A, Estergard, W, Henley, D, \& Sundell, K. (2011). Variability in MMSE scores between screening and baseline visits in 2 large, multi-national Alzheimer's Disease study programs: a concern and potential solution (Paper presented at the 4th Clinical Trials in Alzheimer's Disease Conference, San Diego, USA).

Morris, JC. (1983). The Clinical Dementia Rating (CDR): current version and scoring rules. Neurology, 43, 2412-2414.

Nasreddine, ZS, Phillips, NA, Bédirian, V, Charbonneau, S, Whitehead, V, Collin, I, Cummings, JL, \& Chertkow, H. (2005). The Montreal Cognitive Assessment, MoCA: a brief screening tool for mild cognitive impairment. Journal of the American Geriatric Society, 53(4), 695-699.

Nathan, PJ, Boardley, R, Scott, N, Berges, A, Maruff, P, Sivananthan, T, \& Lai, R. (2013). The safety, tolerability, pharmacokinetics and cognitive effects of GSK239512, a selective histamine $\mathrm{H} 3$ receptor antagonist in patients with mild to moderate Alzheimer's disease: a preliminary investigation. Current Alzheimer Research, 10(240-251), 240.

Norman, KA, \& O'Reilly, RC. (2003). Modeling hippocampal and neocortical contributions to recognition memory: a complementary-learning-systems approach. Psychological review, 110(4), 611-646.

Nuechterlein, KH, Green, MF, Kern, RS, Baade, LE, Barch, DM, Cohen, JD, \& Marder, SR. (2008). The MATRICS Consensus Cognitive Battery, part 1: test selection, reliability, and validity. American Journal of Psychiatry, 165(2), 203-213.

Petersen, RC, Aisen, PS, Beckett, LA, Donohue, MC, Gamst, AC, Harvey, DJ, \& Weiner, MW. (2010). Alzheimer's Disease Neuroimaging Initiative (ADNI): clinical characterization. Neurology, 74, 201-209.

Petersen, RC, Smith, GE, Waring, SC, Ivnik, RJ, Tangalos, EG, \& Kokmen, E. (1999). Mild cognitive impairment: clinical characterization and outcome. Archives of Neurology, 56(3), 303-308

Proust-Lima, C, Amieva, H, Dartigues, JF, \& Jacqmin-Gadda, H. (2007). Sensitivity of four psychometric tests to measure cognitive changes in brain agingpopulation-based studies. American Journal of Epidemiology, 165(3), 344-350.

Rowe, CC, Ellis, KA, Rimajova, M, Bourgeat, P, Pike, KE, Jones, G, \& Villemagne, V. (2010). Amyloid imaging results from the Australian Imaging, Biomarkers and Lifestyle (AIBL) study of aging. Neurobiology of Aging, 31, 1275-1283.

Snaith, RP, \& Zigmond, AS. (1986). The hospital anxiety and depression scale. British Medical Journal, 292, 344.

Snyder, PJ. (2013). The retooling of old cognitive tests as an interim step on the path to validating a next generation of neuropsychological paradigms and assays. Alzheimer's \& Dementia, 9, S1-S3.

Strauss, E, Sherman, EMS, \& Spreen, O. (2006). A compendium of neuropsychological tests: Administration, norms and commentary (3rd ed.). New York: Oxford University Press.

Swets, JA. (1996). Signal detection theory and ROC analysis in psychology and diagnostics: Collected papers. New Jersey: Lawrence Erlbaum Associates.

Villemagne, VL, Burnham, S, Bourgeat, P, Brown, B, Ellis, KA, Salvado, O, \& AIBL Research Group. (2013). Amyloid $\beta$ deposition, neurodegeneration and cognitive decline in sporadic Alzheimer's disease: a prospective cohort study. Lancet Neurology, 12(4), 357-367.
Wechsler, D. (2001). Wechsler Test of Adult Reading: Examiner's manual. San Antonio, TX: The Psychological Corporation.

Winblad, B, Palmer, K, Kivipelto, M, Jelic, V, Fratiglioni, L, Wahlund, LO, \& Petersen, RC. (2004). Mild cognitive impairment - beyond controversies, towards a consensus: report of the International Working Group on mild cognitive impairment. Journal of Internal Medicine, 256(3), 240-246.

Yassa, MA, Stark, SM, Bakker, A, Albert, MS, Gallagher, M, \& Stark, CE. (2010). Highresolution structural and functional MRI of hippocampal CA3 and dentate gyrus in patients with amnestic mild cognitive impairment. Neuro Image, 51(3), 1242-1252

doi:10.1186/2050-7283-1-30

Cite this article as: Maruff et al:: Clinical utility of the cogstate brief battery in identifying cognitive impairment in mild cognitive impairment and Alzheimer's disease. BMC Pharmacology and Toxicology 2013 1:30.

\section{Submit your next manuscript to BioMed Central and take full advantage of:}

- Convenient online submission

- Thorough peer review

- No space constraints or color figure charges

- Immediate publication on acceptance

- Inclusion in PubMed, CAS, Scopus and Google Scholar

- Research which is freely available for redistribution 